Diabetesprädiktion mithilfe des $\mathrm{HbA}_{1 c}$

\title{
Ein Wert von 5,7 Prozent ist schon nicht mehr „normal“
}

\begin{abstract}
Cheng $P$, Neugaard $B$, Foulis $\mathrm{P}$, Conlin PR. Hemoglobin a1c as a predictor of incident diabetes. Diabetes Care;34(3):610-5.
\end{abstract}

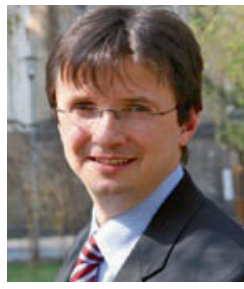

Prof. Dr. med Peter E. H. Schwarz
Fragestellung: Wie potent ist der $\mathrm{HbA}_{1 \mathrm{c}}$ bei der Vorhersage einer Diabeteserkrankung?

Hintergrund: Mit der Einführung des $\mathrm{HbA}_{1 \mathrm{c}}$ als weiteres diagnostisches Kriterium zur Diabetesdiagnose, wird es eine Vielzahl von $\mathrm{HbA}_{1 \mathrm{c}}$-Werten geben, die unterhalb vorgegebener Referenzwertgrenzen liegen. Es wird von uns gefordert werden, ein Risikoassessment für diese $\mathrm{HbA}_{1 \mathrm{c}}$-Werte zu etablieren und vor allem dem Patienten zu beschreiben, welches Risiko er bei einem bestimmten $\mathrm{HbA}_{1 \mathrm{c}}$ hat. Aus diesem Grund ist es notwendig, den $\mathrm{HbA}_{1 c}$-Wert kritisch daraufhin zu prüfen, ob dieser Surrogatparameter für die Glukosetoleranz besser geeignet ist, einen zukünftigen Diabetes zu prognostizieren, als das Messen der Glukosetoleranz selbst. Zusätzlich muss bekannt sein wo der $\mathrm{HbA}_{1 \mathrm{c}}$-Wert Stärken und Schwächen hat. Entscheidend wird dabei sein, wie wir mit mit Werten im Bereich von 5,7-6,5\% umgehen und was das für ein Risikoassessment in der klinischen Praxis bedeutet. Genauso ist es aber entscheidend, auch für $\mathrm{HbA}_{1 \mathrm{c}}$-Werte unter 5,7\% Risikoaussagen treffen zu können. Mit diesem Thema beschäftigt sich der vorliegende Artikel.

Patienten und Methodik: 12589 Patienten wurden auf Basis der klinischen Patientenakten aus zwei Kli- niken der „Veterans Affair Medical Centers“ wegen eines Ausnahme- $\mathrm{HbA}_{1 \mathrm{c}}$ von $<6,5 \%$ zwischen Januar 2000 und Dezember 2001 in die Studie aufgenommen. Patienten mit Diabetes mellitus wurden ausgeschossen. 12375 Patienten hatten später mindestens eine Follow-upVisite. Alle Patienten wurden acht Jahre beobachtet und jene mit einer Diabetesdiagnose identifiziert.

Ergebnisse: Mit einem mittleren Follow-up von 4,4 Jahren entwickelten 3329 Personen (26,9\%) einen Diabetes mellitus. Ein $\mathrm{HbA}_{1 \mathrm{c}}$ von über $5 \%$ beinhaltete ein signifikantes Risiko während der Verlaufszeit einen Diabetes zu entwickeln. Eine $\mathrm{HbA}_{1 \mathrm{c}}$-Erhöhung um 0,5\% in dem Bereich zwischen 5 und 6,4\% bedeutete, bis 5,4\% einer Risikoerhöhung um das 1,7-Fache, bis 5,9\% um das 4,87-Fache und zwischen 6 und 6,4 um das 16,6-Fache. Basierend auf diesen Werten entwickelten die Autoren mithilfe von $\mathrm{HbA}_{1 \mathrm{c}}$, Alter, BMI und systolischem Blutdruck ein Risikoprädiktionsmodell.

Schlussfolgerungen: Die Diabetesinzidenz erhöht sich progressiv, kontinuierlich und signifikant ab einem $\mathrm{HbA}_{1 \mathrm{c}}$ von $5 \%$ und selbst „normale $\mathrm{HbA}_{1 \mathrm{c}}$-Bereiche beinhalten ein substanzielles Risiko. Hochnormaler $\mathrm{HbA}_{1 c}$ ist ein guter Prädiktor für zukünftigen Diabetes und sollte Anlass für Präventionsmaßnahmen sein.
Kommentar: Wie schon öfter wurde hier der $\mathrm{HbA}_{1 \mathrm{c}}$ und seine Fähigkeit, Diabetes vorherzusagen, untersucht. Diese Studie konzentriert sich mehr auf niedrigere $\mathrm{HbA}_{1 \mathrm{c}}$-Werte und ergibt, dass auch in $\mathrm{HbA}_{1 \mathrm{c}}$-Bereichen unter $5,7 \%$ ein substanziell erhöhtes Diabetesrisiko zu finden ist. Diese retrospektive Studie bezog vorwiegend ältere Männer ein, und zwar aus Veteranenkrankenhäusern. Trotz alledem ergab die Untersuchung, dass $\mathrm{HbA}_{1 c}$-Werte unter 5,7 nicht „,normal“ bedeutet.

Was bedeutet das für unsere klinische Praxis und für ein Risikoassessment und eine Risikostratifizierung unserer Patienten?

Nach der vorliegenden Studie birgt ein $\mathrm{HbA}_{1 \mathrm{c}}$ von 5,6 ein signifikantes prospektives Diabetesrisiko. Gleichermaßen ist es nicht vertretbar, auch in niedrigeren Bereichen jetzt jeweils zusätzlich einen oralen Glukosetoleranztest vorzuschreiben und damit bleiben für diese Klientel am Ende eine Beratung und lebensstilorientierte Maßnahmen zur Prävention eines Diabe- tes mellitus. Hier kann unter Umständen der $\mathrm{HbA}_{1 \mathrm{c}}$ in Zukunft sein Potenzial entfalten.

Bei einem $\mathrm{HbA}_{1 c}$ von 5,6 und dem damit verbunden fast fünffach erhöhten Diabetesrisiko, kann das ein Argument für Risikopersonen sein, an einer Lebensstilmaßnahme teilzunehmen. Genauso könnte aber auch ein Schrittzähler eine weitere mögliche Maßnahme in diesem Bereich sein. Wir müssen in den nächsten Monaten weitere Diskussionen führen, was ein „hochnormaler" $\mathrm{HbA}_{1 \mathrm{c}}$-Wert für die Risikostratifizierung in der klinischen Praxis bedeutet.

Prof. Dr. med. Peter E. H. Schwarz

Medizinische Klinik III

Universitätsklinikum Carl Gustav Carus der TU Dresden

Fetscherstr. 74

D-01307 Dresden

Peter.Schwarz@uniklinikum-dresden.de 